\title{
Physical studies of 81P/Wild 2 from the last two apparitions ${ }^{\star}$
}

\author{
Z.-Y. $\operatorname{Lin}^{1,3}$, L. M. Lara ${ }^{1}$ J. B. Vincent ${ }^{2}$, and W.-H. Ip ${ }^{3}$ \\ 1 Instituto de Astrofísica de Andalucía (CSIC), Glorieta de la Astronomía s/n, 18008 Granada, Spain \\ e-mail: zlin@iaa.csic.es \\ 2 Max-Planck-Institut für Sonnensystemforschung, Max-Planck-Strasse 2, 37191 Katlenburg-Lindau, Germany \\ 3 Institute of Astronomy, National Central University, Taiwan \\ Received 8 March 2011 / Accepted 2 November 2011
}

\section{ABSTRACT}

\begin{abstract}
Context. We downloaded data for the comet 81P/Wild 2 for May and June 2004 from the ESO archive and monitored this comet from January to August 2010 during its 6th perihelion passage since its discovery in 1978. Photometric data were used to monitor the comet's gas and dust activity as a function of heliocentric distance. Non-photometric data were used for an analysis of the evolution of the dust coma morphology.

Aims. The goal of the analysis of the observational data was to characterize the evolution of the cometary activity as the nucleus approaches the Sun. We also aimed to assess the gas and dust production rates for the last two apparitions and investigated the evolution of the dust coma morphology during the last passage in 2010.

Methods. The long-slit spectra data were acquired with the EFOSC2 instrument mounted at the 3.6. m ESO telescope in Chile, while narrowband and broadband images were obtained using the 1-m telescope at the Lulin Observatory in Taiwan. Image enhancing techniques were used to investigate the evolution of the dust coma morphology in the R-broadband images, and we modeled the evolution of these dust jets. Where possible, we studied the dust and gas production rate, and the radial profiles of the dust brightness in the Sun-anti-sunward directions.

Results. The morphological analysis helped us to detect the jet structures in the dust coma by using the Larson-Sekanian filter. In May 2004 , only one feature had been found on the sunward side. In 2010, one of the jet features had been switched-off after January, and at least four new jets were found from April to May. The physical properties show an average reddening between $4420 \AA$ and $6840 \AA$ of $8.4 \%$ per $1000 \AA$ and the average resulting $\log$ of the $\mathrm{C}_{2}$-to-CN production rate ratio is $-0.454 \pm 0.13$, which would place Wild 2 in the "depleted" category according to the taxonomic classification. The slope of the radial dependence of the gas production rates for $\mathrm{CN}$ and $\mathrm{C}_{2}$ is very consistent with observations of many other Jupiter-family comets. The slopes of the surface brightness lie between 0.98 and 1.38 in the images taken at the Lulin observatory, whose the range interval from $2000 \mathrm{~km}$ to $30000 \mathrm{~km}$ is roughly consistent with the $\rho^{-1}$ law. However, the gradient of the radial profile in the sunward direction in the outer region $(4.0 \leq \log \rho(\mathrm{km}) \leq 4.5)$ is slightly steeper than that in the inner region $(3.3 \leq \log \rho(\mathrm{km}) \leq 4.0)$.
\end{abstract}

Key words. comets: individual: 81P/Wild 2

\section{Introduction}

Comet 81P/Wild 2 (hereafter Wild 2) is a Jupiter-family comet (JCF) discovered by Paul Wild in early January, 1978. Owing to a close encounter with Jupiter in 1974 at a distance of $0.006 \mathrm{AU}$ from the planet, the orbital parameters changed such that the perihelion distance decreased by $3.5 \mathrm{AU}$, from $4.9 \mathrm{AU}$ to $1.4 \mathrm{AU}$, allowing the cometary surface to receive a higher amount of radiation from the Sun. The orbital period of comet Wild 2 also changed from 57 years to 6.4 years (Sekanina 2003). This relatively young photometric age of $13 \mathrm{cy}$, a parameter related to cometary activity, was estimated by using the secular light curves obtained in the 1990, 1997, and 2003 apparitions (Ferrin 2007). As a consequence of this young age, comet Wild 2 has probably encountered fewer intense heating episodes than other JFCs, therefore we can expect to detect new and pristine material in future apparitions.

Comet Wild 2 was the target of NASA's Stardust mission, with a close encounter on 2 January 2004. The Stardust project was primarily a dust sample return mission whose primary goal

* Based on observations made with the European Southern Observatory telescopes obtained from the ESO/ST-ECF Science Archive Facility. was to collect sub-millimeter particles for laboratory analysis (Brownlee et al. 2004). During its flyby, Stardust also obtained many high-resolution images. These images revealed large numbers of jets projecting around almost the entire perimeter of the nucleus. The highly collimated jets indicate that the source regions on the cometary surface or subsurface were small. This phenomenon was also seen in the comet 19P/Borrelly (Yelle et al. 2004; Soderblom et al. 2004). Unfortunately, the observing conditions of ground-based telescopes during the 2003 perihelion and flyby time were extremely poor, because the comet was in conjunction with the Sun. Under these poor conditions, very little data were obtained at very high airmass (Farnham et al. 2005). In addition, most of the quality data were taken in May and June of 2004.

In 2010, comet Wild 2 was only 0.67 AU distant from Earth. This represented a good chance to obtain relatively good spatial resolution and was an opportunity to study the dust and gas properties in the cometary coma. In addition, we also downloaded Wild 2 data from the ESO archive for May 1 and June 16, 2004, which we compared with the comet's at long heliocentric distance. In this paper, we present the photometric and spectrophotometric results from our analysis of the data acquired during the last two apparitions. 
Table 1. Log of Observations performed at the ESO and Lulin observatories.

\begin{tabular}{lcccccc}
\hline \hline Date & $\begin{array}{c}r_{\mathrm{H}} \\
(\mathrm{AU})\end{array}$ & $\begin{array}{c}\Delta \\
(\mathrm{AU})\end{array}$ & $\begin{array}{c}\text { PsAng } \\
(\mathrm{deg})\end{array}$ & $\begin{array}{c}\alpha \\
(\mathrm{deg})\end{array}$ & $\begin{array}{c}\text { Pixel scale } \\
(\mathrm{km})\end{array}$ & Observatory \\
\hline 2004 & & & & & & \\
May 1 & 2.53 & 2.04 & 259.3 & 22.3 & 464.6 & ESO \\
June 16 & 2.81 & 1.85 & 255.3 & 8.4 & 421.3 & ESO \\
\hline 2010 & & & & & & \\
January 14 & 1.64 & 1.10 & 292.7 & 35.5 & 410.8 & Lulin \\
January 15 & 1.64 & 1.09 & 292.6 & 35.4 & 407.1 & Lulin \\
January 16 & 1.64 & 1.08 & 292.5 & 35.4 & 404.0 & Lulin \\
January 17 & 1.64 & 1.07 & 292.5 & 35.4 & 399.7 & Lulin \\
January 18 & 1.64 & 1.06 & 292.4 & 35.3 & 384.7 & Lulin \\
February 26 & 1.60 & 0.78 & 286.8 & 28.7 & 291.3 & Lulin \\
April 7 & 1.66 & 0.67 & 264.8 & 10.3 & 250.3 & Lulin \\
April 24 & 1.71 & 0.70 & 180.5 & 4.8 & 264.2 & Lulin \\
May 4 & 1.74 & 0.75 & 139.2 & 8.7 & 281.0 & Lulin \\
May 20 & 1.81 & 0.86 & 122.2 & 16.1 & 322.0 & Lulin \\
May 31 & 1.86 & 0.96 & 117.8 & 20.1 & 358.9 & Lulin \\
August 1 & 2.19 & 1.80 & 106.6 & 27.3 & 672.7 & Lulin \\
\hline
\end{tabular}

Notes. $r_{\mathrm{h}}$ and $\Delta$ are the geocentric and heliocentric distances in AU. PsAng is the position of the extended Sun-comet vector, measured from north toward east. $\alpha$ is the phase angle Sun-comet-observer.

\section{Observations and data reduction}

\subsection{Instrumentation}

The first set of observations from 2004 are available worldwide from the European Southern Observatory (ESO) Archive, obtained with the $3.6 \mathrm{~m}$ telescope of the ESO, in La Silla, Chile, using the EFOSC2 instrument $(1030 \times 1030$ pixels, binned pixel size: $0.314^{\prime \prime}$, FOV $\left.5.39^{\prime} \times 5.39^{\prime}\right)$. A spectrograph covering the wavelength ranges from $319 \mathrm{~nm}$ to $1095 \mathrm{~nm}$ at a dispersion of $6.6 \AA /$ pixel was used for this relatively faint comet on May 1 and June 16. The maximum magnitude measured in 2004 was a hundred times less than that in 2010, because of the difference between the heliocentric and geocentric distances. The slit width was 2.0 arcsec and the slit length was 4.1 arcmin with a spatial scale of $0.157^{\prime \prime} /$ pixel. The spatial scale used in this study is $0.314^{\prime \prime} /$ pixel, because of the pixel binning. Notice that the slit used here was oriented along the north-south line, which was nearly perpendicular to the Sun-comet direction.

The second observational program, using the one-meter telescope at Lulin Observatory in Taiwan, was planned from January 14 to August 1, 2010. During this observational period, the comet passed through its perihelion (1.598 AU) on February 22 and made its closest approach to Earth (0.673 AU) on April 5, 2010. The observations were carried out using an Asahi $R$-broadband filter and the narrowband filters of the Rosetta filter set. The specifications of these narrowband filters are described as follows: $\mathrm{CN}(387 / 6 \mathrm{~nm}), \mathrm{C}_{2}(514 / 12 \mathrm{~nm})$, blue continuum $(445 / 4 \mathrm{~nm})$, and red continuum $(687.4 / 6 \mathrm{~nm})$. The camera (PI 1300B) had a pixel scale of $0.516^{\prime \prime}$ and a field of view of $11.2^{\prime} \times 11.6^{\prime}$. Details of our observations of comet Wild 2 are given in Table 1.

\section{2. $C C D$ images and reduction}

The data reduction followed standard procedures. In the outline, the procedure began with bias subtraction in the ESO images and dark-current subtraction in the Lulin images. Then we flatfield corrected of all frames followed by the subtraction of the night sky contribution. For the observations obtained at the ESO in 2004 and the Lulin observatory in 2010, the night sky levels were determined from parts of the cometary images that do not contain contributions from the cometary coma. The extinction coefficients of the narrowband and broadband R-filters were determined for all nights based on the photometric sky conditions, using photometric stars, like Feige 67, observed at various airmasses during the night. These data were used to convert the measured counting rates into physical units; for details see Lin et al. (2007).

\subsection{Spectra and reduction}

The spectrum taken on May 1, 2004, at ESO is shown in Fig. 1 (left-panel). The spectroscopic data were reduced following the standard procedure, including bias and flat-field corrections and cosmic-ray removal. Owing to the relatively faint brightness of comet Wild 2 in 2004, the sky background can be determined by averaging directly at both sides of the spatial directions (Fig. 1, middle-panel). Using this averaged image, the sky-background of the spectrum of comet Wild 2 can be subtracted. Wavelength calibration was performed based on helium-argon lamps exposed at both the beginning and the end of the observations every night, after which the atmospheric extinction effect (using the standard extinction curve for La Silla) was corrected. Finally, flux calibration of each spectrum was conducted based on observations of the spectro-phometric flux standard star LTT7987 (Hamuy et al. 1992) as a flux calibrator in the range of $330 \mathrm{~nm}$ $\sim 1000 \mathrm{~nm}$.

\section{Data analysis and results}

\subsection{Gas production rate}

In order to determine the gas production rates from cometary frames in 2010, the mean radial emission profiles of $\mathrm{CN}$ and $\mathrm{C}_{2}$ were derived from the images with the continuum subtracted (Lin et al. 2009). We fitted the mean radial brightness profiles of $\mathrm{CN}$ and $\mathrm{C}_{2}$ using the Haser model (Haser 1957), which describes the isotropic emission of cometary neutral molecules and their daughter molecules and radicals. The parameter used for the parent velocity is $v_{\mathrm{p}}=0.85 r^{-0.5} \mathrm{~km} \mathrm{~s}^{-1}$ (Fray et al. 2005) and for the daughter velocity it is $1 \mathrm{~km} \mathrm{~s}^{-1}$. The fluorescence 

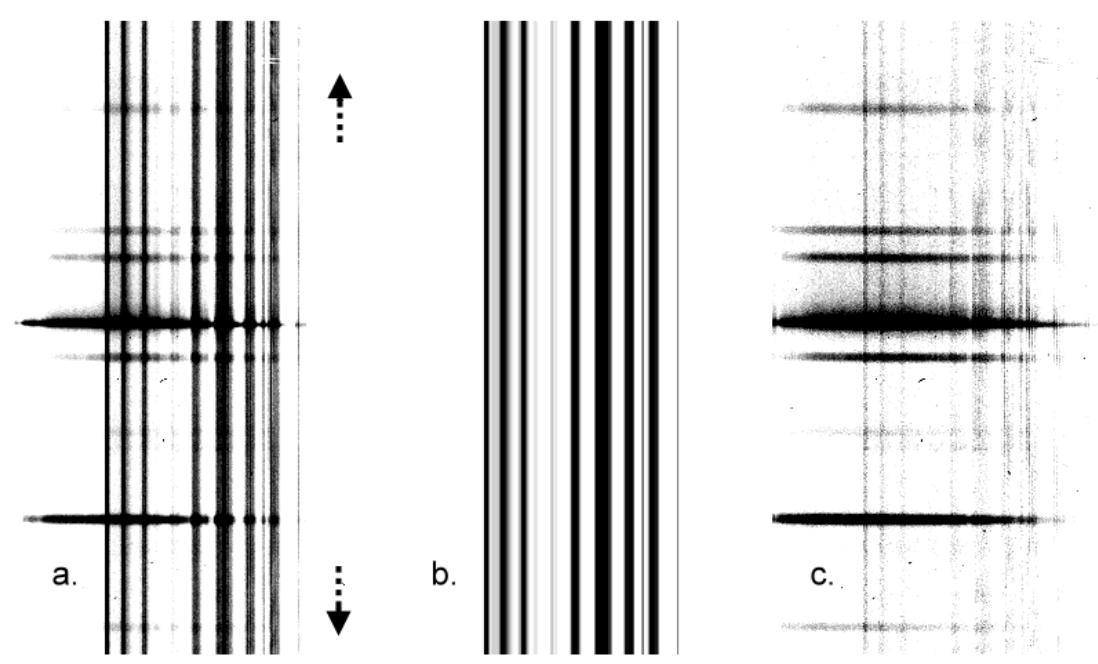

Fig. 1. Spectrum of comet 81P/Wild 2 with sky-background reduction and subtraction: a) the spectrum reduced for bias and flat. The region of the sky-background can be measured directly at both sides (arrows) of the spectrum. b) The average sky-background spectrum. c) The cometary spectrum with the sky-background subtraction.

Table 2. Emission and continuum bands extracted from the spectra.

\begin{tabular}{lccc}
\hline \hline $\begin{array}{l}\text { Spectral } \\
\text { region }(\AA)\end{array}$ & Species & $\begin{array}{c}\text { Blue-side } \\
\text { continuum }(\AA)\end{array}$ & $\begin{array}{c}\text { Red-side } \\
\text { continuum }(\AA)\end{array}$ \\
\hline $3830-3950$ & $\mathrm{CN}$ & $3700-3815$ & $3910-3970$ \\
$4860-5185$ & $\mathrm{C}_{2}$ & $4780-4850$ & $5195-5295$ \\
\hline
\end{tabular}

efficiency factors (g-factors) of $\mathrm{CN}$ are $3.1 \times 10^{-13}$ in January and $4.2 \times 10^{-13}$ in May (Schleicher et al. 2010) and $C_{2}$ in January and May are $4.5 \times 10^{-13}$ (A'Hearn et al. 1995). The scale-lengths used here for $r_{\mathrm{H}}$ at $1 \mathrm{AU}, l_{\mathrm{p}}$ and $l_{\mathrm{d}}$, are $1.3 \times 10^{4} \mathrm{~km}, 2.1 \times 10^{5} \mathrm{~km}$ for $\mathrm{CN}$ and $2.2 \times 10^{4} \mathrm{~km}, 6.6 \times 10^{4} \mathrm{~km}$ for $\mathrm{C}_{2}$, respectively (A'Hearn et al. 1995). The results indicated in Fig. 2 with opencircle symbols.

From the spectra obtained in 2004, the spectral range of $\mathrm{CN}$, $\mathrm{C}_{2}$ and the continuum at both sides of the gas emission are specified and listed in Table 2 (Lara et al. 2001). Here, we used the red- and blue-side continuum for linear fitting, and based on the fitting results subtracted the underlying continuum in the gas emission bands. After subtraction, we integrated the spectral regions for Haser profile fitting. The g-factor used in the spectra of $\mathrm{CN}$ is $4.1 \times 10^{-13}$ (Schleicher 2010) and for $\mathrm{C}_{2}$ it is $4.5 \times 10^{-13}$ (A'Hearn et al. 1995). The results of the production rate in these two apparitions are also indicated by the open triangles in Fig. 2.

In Fig. 2 we plot the logarithm of the production rates for $\mathrm{CN}$ and $\mathrm{C}_{2}$ as a function of the heliocentric distance $\left(r_{\mathrm{H}}\right)$. The data points including those obtained by Farnham et al. (2005) for pre-perihelion and post-perihelion observations and the ESO and Lulin results are presented here. There are three implicit assumptions made in Fig. 2 with multiple apparitions: there are no secular variations from one apparition to another, the Haser scale lengths are nearly the same, and the rotational variations in the production rates are small. We used the linear fitting to estimate the slope of the radial dependence of the gas production rate, $Q \sim r^{\alpha}$ (Fig. 2, dashed line), and the slopes ( $\alpha$ ) of $\mathrm{CN}$ and $\mathrm{C}_{2}$ are -3.68 and -4.39 , respectively. The measurements of gas and dust production rate in 1978 were significantly higher than for the other apparitions because of the evolution effect. This effect was associated with Wild 2's first approach to the Sun after its orbit was changed in 1974. (Farnham et al. 2005). We therefore discarded the data taken by Farnham in 1978 (opened squares in Fig. 2). The slopes of $\mathrm{CN}$ and $\mathrm{C}_{2}$ would consequently change from -3.68 to -2.58 and from -4.39 to -2.60 , respectively (Fig. 2, solid-line). This result is completely consistent with the observations of many other Jupiter-family comets, i.e. $Q($ gas $) \sim r_{\mathrm{H}}{ }^{-2.7}$ (A'Hearn et al. 1995).

\subsection{Composition}

The mean value in the $\log \left[Q\left(\mathrm{C}_{2}\right) / Q(\mathrm{CN})\right]$ for typical comets is approximately +0.13 and for depleted comets approximately -0.11 in A'Hearn et al. (1995). The average value in this work (Table 3 ) is $-0.45 \pm 0.13$, which would place Wild 2 in the "depleted" category according to this taxonomic classification. However, the value is about four times lower than the mean value for depleted comets but is similar to the average value $(-0.58 \pm 0.15)$ estimated by Fink et al. (1999), which was measured to be $(-0.34 \pm 0.17)$ by Farnham et al. (2005). The data of $\log \left[Q\left(\mathrm{C}_{2}\right) / Q(\mathrm{CN})\right]$ for the interval from 1978 to 2010 is plotted in Fig. 3.

\subsection{Dust coma properties}

We used the Af $\rho$ parameter (A'Hearn et al. 1984) to characterize the dust activity of Wild 2 . The derived values for January to August 2010 at several cometocentric distances are presented in Table 4. The derived Af $\rho$ values for the narrowband filter can be taken to estimate the color of the cometary dust (Jewitt \& Meech 1986) as the normalized gradient of the Af $\rho$ product between the blue (BC, $\lambda_{0}=4430 \AA$ ) and red ( $\mathrm{RC}, \lambda_{0}=6840 \AA$ ) continuum filters. The dust colors can be converted to percentage of reddening per $1000 \AA$, allowing for the different locations of the band passes between the two filters sets, and is defined by the following relation:

color $=\frac{R C_{\mathrm{Af} \rho}-B C_{\mathrm{Af} \rho}}{6840-4430} \frac{2000}{R C_{\mathrm{Af} \rho}+B C_{\mathrm{Af} \rho}}$.

The color examined between the blue- and red-continuum filters on January 15 and May 4 is $8.2 \%$ and $8.6 \%$, respectively. These values agree with those presented by Schulz et al. (2003) 

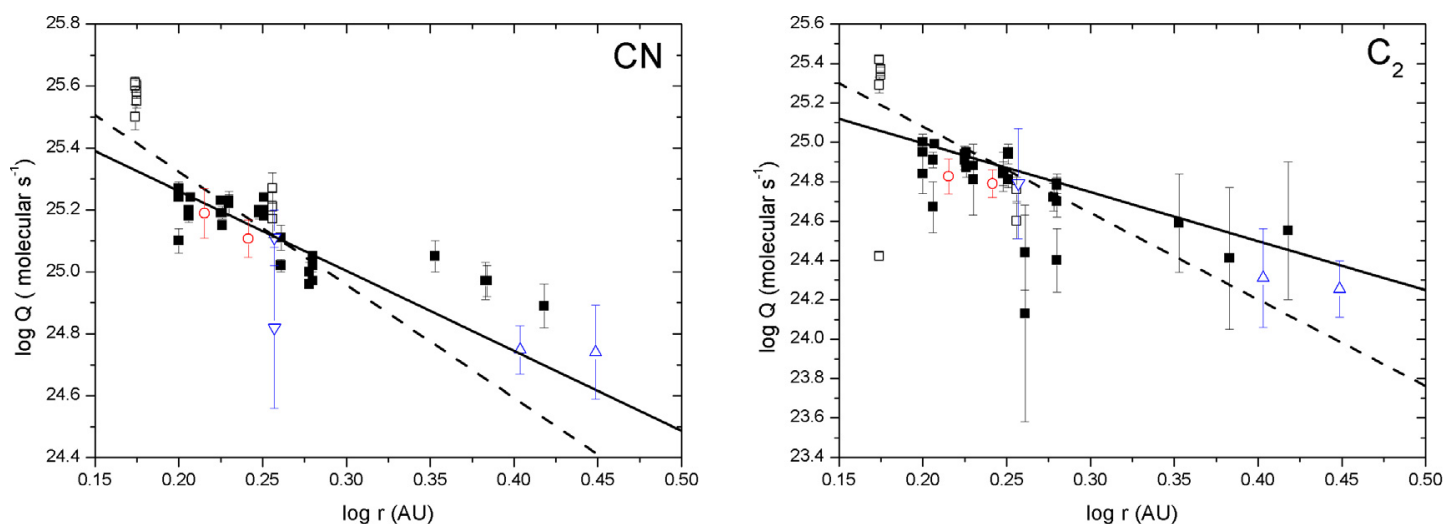

Fig. 2. $\mathrm{Log}$ of production rates for $\mathrm{CN}($ left $)$ and $\mathrm{C}_{2}$ (right) plotted as a function of the log of the heliocentric distance. Different symbols come from different data sets; opened square, filled symbols and opened inverse-triangle are the data from four apparitions (1978 2003) taken by Farnham et al. (2005). The open circle and triangle symbols are the data taken from the Lulin observatory in 2010 and from La Silla in 2004, respectively. Notice that the data from $2003 \sim 2004$ are all represented by triangle symbols. Two different results of the linear fitting are present. The dash line is representative of all data obtained from 1978 to 2010 and the solid line is for all data obtained from 1983 to 2010.

Table 3. The results and ratio of gas production rates.

\begin{tabular}{lccc}
\hline \hline & $\mathrm{CN}$ & $\mathrm{C}_{2}$ & $\log \left[Q\left(\mathrm{C}_{2}\right) / Q(\mathrm{CN})\right]$ \\
Date & $Q\left(10^{24}\right.$ molecules/s $)$ & $Q\left(10^{24}\right.$ molecules/s $)$ & \\
\hline 2004 May 1 & $5.6 \pm 1.1$ & $2.0 \pm 1.6$ & -0.447 \\
2004 June 16 & $5.5 \pm 2.3$ & $1.8 \pm 0.7$ & -0.482 \\
\hline 2010 Jan. 15 & $15.5 \pm 2.1$ & $3.8 \pm 0.5$ & -0.607 \\
2010 May 4 & $5.9 \pm 1.0$ & $3.1 \pm 0.5$ & -0.280 \\
\hline
\end{tabular}

Table 4. Dust coma properties.

\begin{tabular}{lccccccc}
\hline \hline Date & $\begin{array}{c}r_{\mathrm{H}} \\
(\mathrm{AU})\end{array}$ & $\begin{array}{c}\alpha \\
(\mathrm{deg})\end{array}$ & $\begin{array}{c}\mathrm{Af}^{a}{ }^{a} \\
(\mathrm{~cm})\end{array}$ & $\begin{array}{c}\mathrm{Af}^{b} \\
(\mathrm{~cm})\end{array}$ & $\begin{array}{c}\text { Slope }(-\mathrm{m}) \\
(3.3 \leq \log \rho \leq 4.5)\end{array}$ & $(3.3 \leq \log \rho \leq 4.0)$ & $(4.0 \leq \log \rho \leq 4.5)$ \\
\hline 2004 (ESO) & & & & & & & \\
May 1 & 2.53 & 22.3 & & & $1.71^{c}, 1.31^{d}$ & $1.62^{c}, 1.40^{d}$ & $1.47^{c}, 0.98^{d}$ \\
June 16 & 3.81 & 1.85 & & & $1.74^{c}, 1.07^{d}$ & $1.71^{c}, 1.22^{d}$ & $1.99^{c}, 1.01^{d}$ \\
\hline 2010 (Lulin) & & & & & & & \\
January 14 & 1.64 & 35.5 & 868.4 & 782.8 & $2.08^{c} ; 1.02^{d}$ & $1.27^{c} ; 1.08^{d}$ & $3.38^{c} ; 0.99^{d}$ \\
January 15 & 1.64 & 35.4 & 566.3 & 538.1 & $1.38^{c} ; 0.99^{d}$ & $1.29^{c} ; 1.05^{d}$ & $1.55^{c} ; 0.97^{d}$ \\
January 16 & 1.64 & 35.4 & 566.2 & 534.8 & $1.34^{c} ; 0.99^{d}$ & $1.26^{c} ; 1.03^{d}$ & $1.51^{c} ; 0.95^{d}$ \\
January 17 & 1.64 & 35.4 & 613.3 & 573.1 & $1.37^{c} ; 0.98^{d}$ & $1.34^{c} ; 1.04^{d}$ & $1.46^{c} ; 0.96^{d}$ \\
January 18 & 1.64 & 35.3 & 608.3 & 570.9 & $1.38^{c} ; 1.04^{d}$ & $1.21^{c} ; 1.10^{d}$ & $1.63^{c} ; 0.97^{d}$ \\
February 26 & 1.60 & 28.7 & & & $1.21^{c} ; 1.04^{d}$ & $1.13^{c} ; 1.13^{d}$ & $1.46^{c} ; 0.99^{d}$ \\
April 7 & 1.66 & 10.3 & & & $1.13^{c} ; 1.17^{d}$ & $1.20^{c} ; 1.14^{d}$ & $1.24^{c} ; 1.22^{d}$ \\
April 24 & 1.71 & 4.8 & 604.2 & 531.6 & $1.23^{c} ; 1.23^{d}$ & $1.27^{c} ; 1.19^{d}$ & $1.13^{c} ; 1.27^{d}$ \\
May 4 & 1.74 & 8.7 & 569.9 & 532.0 & $1.06^{c} ; 1.19^{d}$ & $1.10^{c} ; 1.13^{d}$ & $1.01^{c} ; 1.27^{d}$ \\
May 20 & 1.81 & 16.1 & & & $1.12^{c} ; 1.16^{d}$ & $1.17^{c} ; 1.09^{d}$ & $1.02^{c} ; 1.28^{d}$ \\
May 31 & 1.86 & 20.1 & & & $1.09^{c} ; 1.17^{d}$ & $1.17^{c} ; 1.10^{d}$ & $1.03^{c} ; 1.30^{d}$ \\
August 1 & 2.19 & 27.3 & 192.3 & 195.0 & $1.09^{c} ; 1.18^{d}$ & $1.23^{c} ; 1.10^{d}$ & $1.01^{c} ; 1.42^{d}$ \\
\hline
\end{tabular}

Notes. Af $\rho$ values are derived for a photometric circular aperture. ${ }^{(a)}$ The radius in aperture is $10000 \mathrm{~km} ;{ }^{(b)}$ the radius in aperture is $20000 \mathrm{~km} ;{ }^{(c)}$ is measured from the sunward direction; ${ }^{(d)}$ is refers to tailward direction. Errors estimated here are $5 \%$ for the Af $\rho$ and $4 \%$ in slopes, respectively.

from broadband BVR images and those found by Farnham et al.

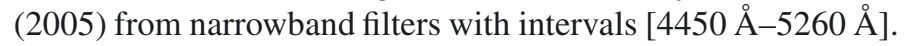

\subsection{Dust brightness profiles}

To analyze the overall brightness distribution of the coma, onedimensional profiles were obtained directly from the Sun-tail direction. In the double logarithmic representation, the radial coma profiles should be straight lines with a slope of $m=-1$ (Jewitt \& Meech 1987) under a steady-state situation. Our measurements, based on the ESO 2004 and Lulin 2010 observations, are listed in Table 4 . The slopes lie between 0.98 and 1.38 with a range interval from $2000 \mathrm{~km}$ to $30000 \mathrm{~km}$ and are roughly consistent with the $\rho^{-1}$ law ( $\rho$ is the projected radial distance from the nucleus in the plane of the sky). The slopes in the sunward direction for January 14, 2010, are also drived.

The slope on January 14, 2010, shows an interesting kneelike feature in the sunward direction (Fig. 4), with a slope of 1.27 in the inner region $[2000 \mathrm{~km} \sim 10000 \mathrm{~km}$ ] and 3.38 in the outer region $[10000 \mathrm{~km} \sim 30000 \mathrm{~km}]$. These steeper slopes may be caused by non-steady state dust emission or possibly by dust grain fading or destruction (Baum et al. 1992). If this is the case, the dust grains associated with high Af $\rho$ (Table 4) could have come from an earlier outburst or other event. Unfortunately, no observations are available before January 14, which prevents a conclusion about whether we witnessed an outburst or if there 


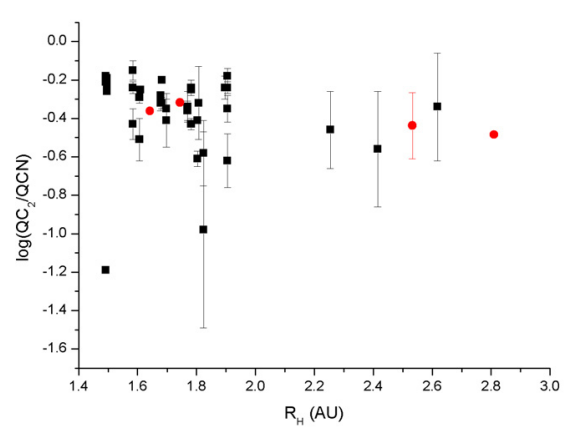

Fig. 3. $\log Q\left(\mathrm{C}_{2}\right)$ to $Q(\mathrm{CN})$ ratio plotted as a function of the heliocentric distance. The filled circles represent the estimates of the present work and the filled squares indicate the results obtained by Farnham et al. (2005).

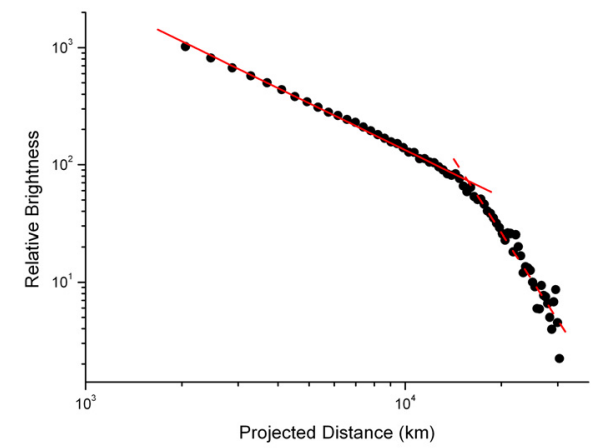

Fig. 4. Radial brightness profile, $\log B$, vs. projected distance, $\log \rho$ on January 14,2010 . The slope of the sunward direction shows a very different knee-like phenomenon, wiht a slope 1.27 (solid line) in the inner region $[2000 \mathrm{~km} \sim 10000 \mathrm{~km}]$ and 3.38 (dashed line) in the outer region $[10000 \mathrm{~km} \sim 30000 \mathrm{~km}]$.

was some earlier event. However, if we obtain the profile in the tailward direction, the results in the inner and outer tailward region and in the inner region are consistent with the $\rho^{-1}$ law.

Apart from this interesting feature on January 14, we find that the gradient on January and February 2010 in the outer sunward region is also steeper than that in the inner sunward region. These steep profiles could be influenced by one or more factors: radiation pressure acting on the dust, or temporal changes in the optical properties of the grains, i.e. the sublimation of the ice grains (Jewitt \& Meech 1987), changing either their size or albedo.

\section{Morphology of coma structures}

\subsection{Morphology}

We describe the morphology and evolution of coma structures acquired during the last two apparitions. During the period in 2004 , the nucleus showed less activity because of the long heliocentric distance. In contrast, the nucleus displayed a lot of activity in the last apparition between January 14 and August 1, 2010 , characterized by the presence of dust jets in the coma. These structures can be treated with routine procedures. Here we applied two independent methods: (1) Adaptive Laplace filter (Böhnhardt \& Birkle 1994); and (2) Larson-Sekanina algorithm (Larson \& Sekanina 1984). The first filter applies a
Laplacian kernel to a logarithmized image, which can remove all gradual variations in linear and second order gradients. Adaptive filtering means to look for the spatial frequency band containing the relevant signal, especially to enhance particularly the structure in this band. Here we applied the MIDAS procedure FILTER/ADAPTIVE with the Laplace filter with the parameters chosen after extensive tests. The second technique is a direct subtraction between the rotated/shifted frame and the original frame. The results of the Larson-Sekanina filtered (angle $=10$ degree) can be found in Fig. 5 .

In case of doubt, we used additional techniques, such as mean-median profile subtraction or unsharp masking to clearly separate morphological features from artifacts. We also made a comparison with the processed images and isophotes of the original images (Fig. 6). Since the contribution of light from gas and ion emissions is small in the $R$-band, we interpret the observed structures as dust reflecting sunlight. We can therefore clearly identify the dust tail and coma fan structures produced by active dust-emitting sources on the rotating nucleus, as described by Sekanina (1987).

In addition to the dust tail (labeled $T$ in Fig. 5) we identified up to seven different dust structures including two sub-structures in our images, as summarized in Table 5. Notice that the position of the coma structures in Table 5 does not correspond to the values that we found in the active regions located at the nucleus. From 14 to 18 January, 2010, a broad northeast jet, which can be divided into two substructures and a narrow southeast jet (labeled $A, B$, and $C$ in Fig. 5) were detected in the solar direction. The difference between substructure A and B is the curvature feature. Substructure A extends first toward the Sun before bending in the tail direction. The apparent broadening away from the nucleus could be caused by the size-sorting of grains through radiation pressure. Substructure B and structure $\mathrm{C}$ do not display the same curvature, although this is very likely a projection effect. Notice that substructure B is very close to substructure $\mathrm{A}$ in the images and as the jet broadens when particles move away from the nucleus, the bent part of structure B cannot be distinguished from structure A. In the image taken on 26 February, only substructures A and B (northeast jet) are detected. Because we have a very good signal-to-noise ratio in these images, the disappearance of structure $\mathrm{C}$ is not a bias in our observations but rather indicates a change in activity, probably the switching-off of one active region. Notice that the Earth-comet observing geometry in January and February remained relatively constant. From early April to late May 2010, at least four new jets appear in addition to substructures A and B (labeled $D, E$, $F, G$ in Fig. 5). There were three new identifiable jets in April and one new additional jet was found at PA 8 degrees in May. The last image in May suffers from the low signal/noise ratio so the structures can barely be noticed, although they might still be present. In August, only one jet can be found in the inner coma, which might have been present all the time from May to August, because their morphology and the orbital configuration did not change during these months.

Images obtained in 2004 are not included in Fig. 5 and Table 5. There were only a few usable images from that period because of the poor tracking. However, we were lucky to find the feature in the images we have. In Fig. 7 we can see the features processed in the dust tail, extending to the southwest, and the jet feature, bending to the south. Unfortunately, the image does not reveal how many features there were in the sunward direction owing to the low signal/noise ratio. 


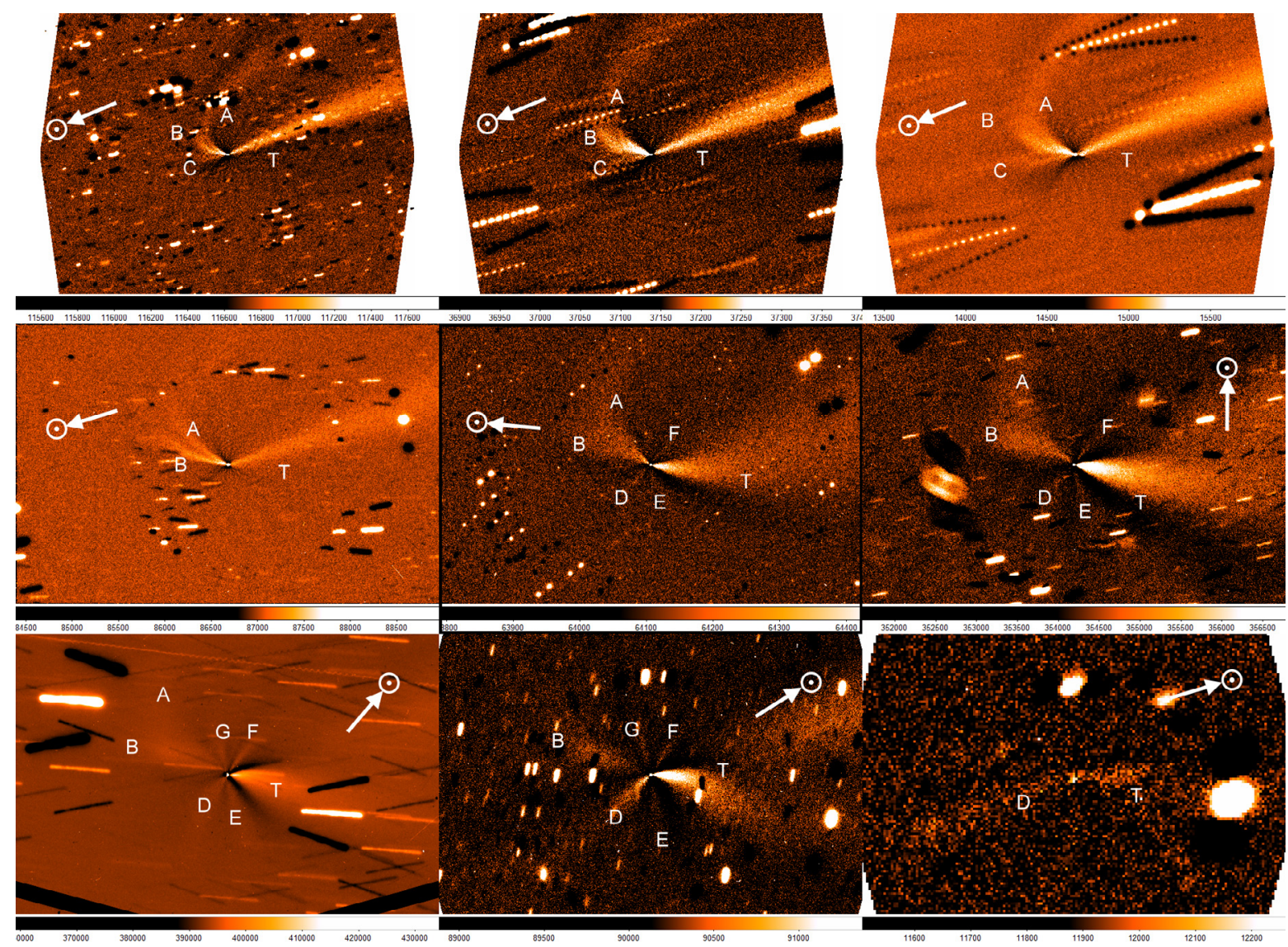

Fig. 5. Images after processing by the Larson-Sekanina algorithm taken on January 14, 17 and 18, (top, from left to right) February 26, April 7, 24 (middle, from left to right) May 4, May 20, August 1 (bottom, from left to right). In all images, north is up, east is to the left. The field of view is $5.85^{\prime} \times 3.87^{\prime}$. The arrow points in the solar projection direction. Structures are identified with capital letters, and T marks the position of the dust tail. All images were obtained with a broadband $R$-filter.
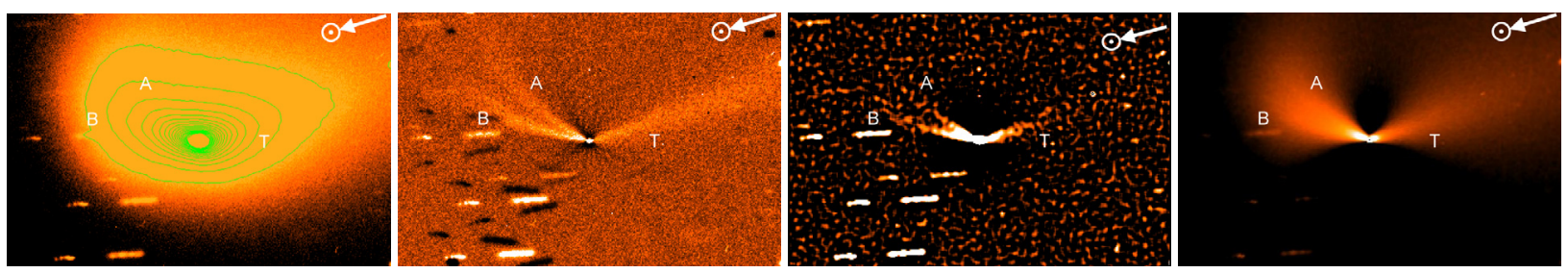

Fig. 6. Isophote of the original image, Larson-Sekanina filtered, adaptive-laplace filtered and divided by mean radial profile (from left to right) images of comet 81P/Wild2 displaying several coma structures on February 26, 2010. Structures are identified with capital letters, T marks the position of the dust tail. North is up, East is to the left., the field of view is $2.92^{\prime} \times 1.94^{\prime}$

Table 5. Position of structures in the dust coma in 2010.

\begin{tabular}{lcccccccccc}
\hline \hline Date & $r_{\mathrm{H}}(\mathrm{AU})$ & $\Delta(\mathrm{AU})$ & $\mathrm{PsAng}\left({ }^{\circ}\right)$ & $\mathrm{A}\left(^{\circ}\right)$ & $\mathrm{B}\left(^{\circ}\right)$ & $\mathrm{C}\left({ }^{\circ}\right)$ & $\mathrm{D}\left({ }^{\circ}\right)$ & $\mathrm{E}\left({ }^{\circ}\right)$ & $\mathrm{F}\left({ }^{\circ}\right)$ & $\mathrm{G}\left({ }^{\circ}\right)$ \\
\hline Jan.-14 & 1.64 & 1.10 & 292.7 & 55 & 80 & 105 & - & - & - & - \\
Jan.-15 & 1.64 & 1.09 & 292.6 & 55 & 80 & 105 & - & - & - & - \\
Jan.-16 & 1.64 & 1.08 & 292.5 & 55 & 80 & 105 & - & - & - & - \\
Jan.-17 & 1.63 & 1.07 & 292.5 & 55 & 80 & 105 & - & - & - & - \\
Jan.-18 & 1.63 & 1.06 & 292.4 & 55 & 80 & 105 & - & - & - & - \\
Feb.-26 & 1.59 & 0.78 & 286.8 & 55 & 80 & - & - & - & - & - \\
Apr.-07 & 1.65 & 0.67 & 264.8 & 40 & 67 & - & 140 & 200 & 320 & - \\
Apr.-24 & 1.70 & 0.70 & 180.5 & 40 & 67 & - & 140 & 200 & 328 & - \\
May-04 & 1.74 & 0.74 & 139.2 & 43 & 69 & - & 140 & 200 & 320 & 8 \\
May-20 & 1.80 & 0.85 & 122.2 & - & 65 & - & 130 & 200 & 328 & 8 \\
May-31 & 1.85 & 0.95 & 117.8 & - & - & - & 130 & - & - & - \\
Aug.-01 & 2.19 & 1.80 & 106.6 & - & - & - & 120 & - & - & - \\
\hline
\end{tabular}

Notes. Identification of the coma structures as shown in Fig. 5. The given position angles refer to the center lines of the structures and may have a maximuma uncertainty of $\pm 10^{\circ}$. North $=0^{\circ}$, East $=+90^{\circ}$. PsAng is the position of the extended Sun-comet vector. The dash indicates that the structure was not detected. 


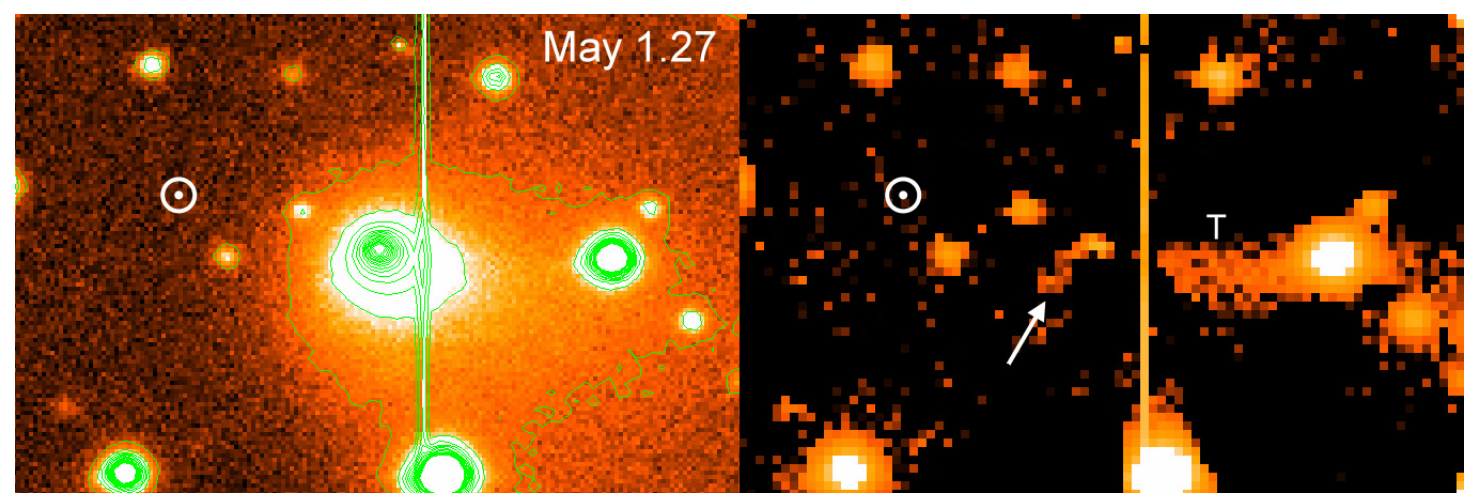

Fig. 7. Left panel: the isophote of the original image taken on May 1, 2004; right panel: same image after image processing by the Larson-Sekanina algorithm. To increase the signal-to-noise ratio, we used a binnned image in the right panel. Besides the dust tail, the jet feature was also detected. North is up, east is to the left. The field of view is $53^{\prime \prime} \times 35^{\prime \prime}$.
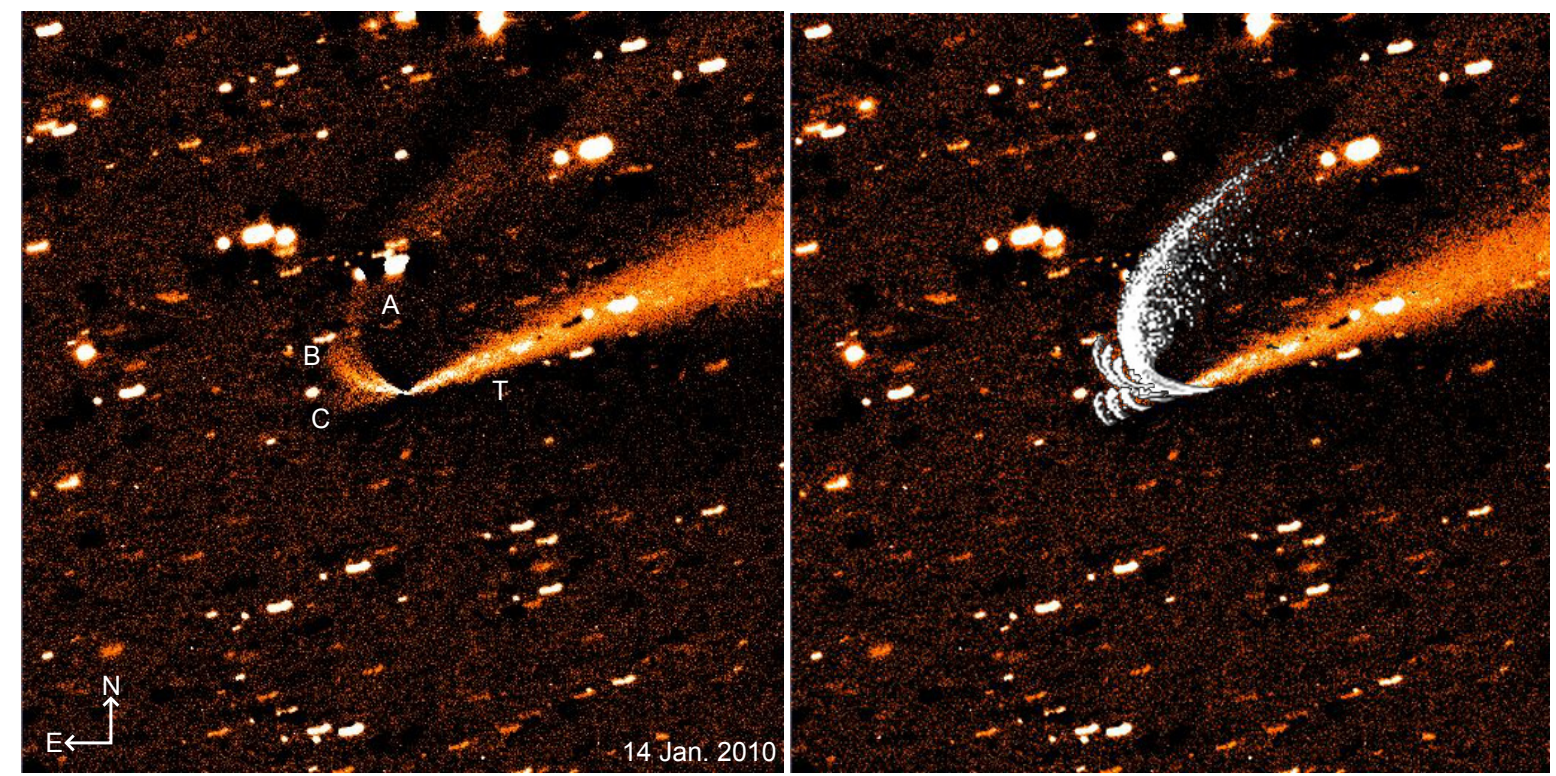

Fig. 8. Left panel: filtered image of comet 81P/Wild 2 showing the presence of dust coma structures on January 14, 2010; right panel: same image with simulated structures as a white overlay.

\subsection{Modeling and interpretation}

The fact that we detected several dust coma structures in 2010 does not come as a surprise; comet $81 \mathrm{P} /$ Wild 2 is known to be very active with many jets and active regions that were observed during its encounter with the spacecraft Stardust in January 2004 (Sekanina 2004). We aimed to investigate whether we were able to use our observations to localize the sources of activity at the surface of the nucleus, and determine whether there had been any changes with respect to what was observed in situ by Stardust six years ago. The analysis was made with the simulation code presented in Vincent et al. (2010), which creates images of dust jets emitted from discreet active regions at the surface of a three dimensional shape model of a cometary nucleus. We first used an ellipsoid to represent the nucleus, and then we refined the analysis by using a more complex model (Farnham et al. 2005). We did not infer the orientation of the spin axis from our images, but instead used the latest value of RA: $110 \pm 3^{\circ}$ and Dec: $-13 \pm 3^{\circ}$ published by Duxbury et al. (2004).

Figure 8 shows an example of a simulation for an image acquired on January 14, 2010. In order to reproduce the coma structures, we needed three different active regions at latitudes of $-30^{\circ},-10^{\circ}$, and $+30^{\circ}$, emitting particles ranging in size from $\mu \mathrm{m}$ to a few tenths of $\mu \mathrm{m}$. The activity is restrained to the equatorial region of the nucleus, and this result agrees with the localization of sources by Sekanina (2004) from the images obtained by Stardust. These three regions are active only during the day, any night-time activity during this epoch would produce different patterns than the observed ones. The switch-off of jet $\mathrm{C}$ after January 2010 is not connected to a change in illumination conditions at the surface, but could be explained for instance by the depletion in sublimating material in the associated active region. From April to the end of May 2010, many more dust structures were detected, and can be explained by the presence of new active regions in the same equatorial region.

\section{Summary}

The jet features of comet $81 \mathrm{P} /$ Wild 2 have been detected during the last two apparitions. The large $\Delta(\sim 2 \mathrm{AU})$ and long perihelion distance $(\sim 2.5 \mathrm{AU})$ in 2004 might be the reason why we cannot detect any more jet features for this active comet. In addition, we observed the switch-off of jet C after January 2010. This phenomenon can be explained by the depletion of the sublimating material in the associated active region. Unfortunately, we did not have the Af $\rho$ variations in between these two months 
because of the bad weather conditions in February. From April to the end of May 2010, many more dust structures were detected, and can be explained by the presence of new active regions in the same equatorial region.

We find that the dust brightness radial profile and dust production rate (Table 4) for January 14 are very distinct, with Af $\rho$ being higher in a factor of 1.5 compared to other days. Unfortunately, no observations are available before January 14, so we are unable to draw a conclusion about whether we witnessed an outburst or if there was some other earlier event. Aside from this date, as can be seen in the images taken at the Lulin observatory, the slopes lie between 0.98 and 1.38, with a range interval from $2000 \mathrm{~km}$ to $30000 \mathrm{~km}$, and are roughly consistent with a $\rho^{-1}$ law. However, the gradient of the radial profile for the sunward direction in the outer region $(4.0 \leq \log$ $\rho(\mathrm{km}) \leq 4.5)$ is slightly steeper than that in the inner region $(3.3 \leq \log \rho(\mathrm{km}) \leq 4.0)$. These steeper profiles could be caused by the effect of the radiation pressure, which acts on the dust when considering the longer cometocentric distance $\rho$.

The average color estimated from the imaging is very consistent with the results from former apparitions. In addition, the resulting $\log$ of the $\mathrm{C}_{2}$-to- $\mathrm{CN}$ production rate ratio is $-0.454 \pm 0.13$, from which is inferred Wild 2 belongs to the depleted class. The slope of the radial dependence of the gas production rates for $\mathrm{CN}(-2.58)$ and $\mathrm{C}_{2}(-2.60)$ is very consistent with those from observations of many other Jupiter-family comets $\left(Q(\right.$ gas $\left.) \sim r_{\mathrm{H}}{ }^{-2.7}\right)$ if we ignore the 1978 data.

Acknowledgements. This work was based on observations obtained at Taiwan's Lulin Observatory. We thank the staff members and Lijing Huang for their assistances with the observations. A part of work is based on observations made with the European Southern Observatory telescopes obtained from the ESO/ST-ECF Science Archive Facility. The research was supported by project
AyA2009-08011 of the Ministerio de Ciencia e Innovacin. Zhong Yi Lin acknowledges a post-doctoral grant awarded by the Junta de Andaluca through project number P07-TIC-274. This work was also supported by grant number NSC 99-2923-M-008-002-MY3 of the Formosa Program.

\section{References}

A'Hearn, M. F., Schleicher, D. G., Millis, R. L., Feldman, P. D., \& Thompson, D. T. 1984, AJ, 89,579

A'Hearn, M. F., Millis, R. L., Schleicher, D. G., Osip, D. J., \& Birch, P. V. 1995, Icarus, 118, 223

Baum, W. A., Kreidl, T. J., \& Schleicher, D. G. 1992, AJ, 104, 1216

Böhnhardt, H., \& Birkle, K. 1994, A\&A, 107, 101

Brownlee, D. E., Horz, F., Newburn, R. L., et al. 2004, Science, 304, 1764

Duxbury, T. C., Newburn, R. L., \& Brownlee, D. E. 2004, JGR, 109

Farnham, T. L., \& Schleicher, D. G. 2005, Icarus, 173, 533

Ferrin, I. 2007, Icarus, 191, 22

Fink, U., Hicks, M. P., \& Fevig, R. A. 1999, Icarus, 141, 331

Fray, N., Bnilan, Y., Cottin, H., Gazeau, M.-C., \& Crovisier, J. 2005, P\&SS, 53, 1243

Jewitt, D. C., \& Meech, K. J. 1987, ApJ, 317, 992

Hamuy, M., Walker, A. R., Suntzeff, N. B., et al. 1992, PASP, 104, 533

Haser, L. 1957, Bull. Soc. Roy. des Sci. Leige, 43, 740

Kurucz, R. L., Furenlid, I., Brault, J., \& Testerman, L. 1984, N. S. O. Atlas No. 1

Lara, L. M., Schulz, R., Stüwe, J. A., \& Tozzi, G. P. 2001, Icarus, 150, 124

Larson, S. M., \& Sekanina, Z. 1984, AJ, 89, 571

Lin, Z. Y., Weiler, M., Rauer, H., \& Ip, W. H. 2007, A\&A, 469, 771

Lin, Z. Y., Lin, C. H., Ip, W. H., \& Lara, L. M. 2009, AJ, 138, 625

Sekanina, Z. 1987, ESA SP-278, 315

Sekanina, Z. 2003, 108, SRD 2-1

Sekanina, Z., Brownlee, D. E., Economou, T. E., Tuzzolino, A. J., \& Green, S. F. 2004, Science, 304, 1769

Schleicher, D. G. 2010, AJ, 140, 973

Schulz, R., Stüwe, J. A., Böhnhardt, H., Gaessler, W., \& Tozzi, G. P. 2003, A\&A, 398,345

Soderblom, L. A., Boice, D. C., Britt, D. T., et al. 2004, Icarus, 167, 4

Yelle, R. V., Soderblom, L. A., \& Jokipii, J. R. 2004, Icarus, 167, 30

Vincent, J.-B., Böhnhardt, H., \& Lara, L.-M. 2010, A\&A, 512, A60 